\title{
Aufbereitung und Veredlung von Kohlenstoff-Vorkonzentraten aus Pyrolyse-Prozessen zu marktfähigen Kohlenstoff-Produkten im Hinblick auf große Oberflächen und hohe Reinheiten
}

\section{Vorstellung des Dissertationsprojektes}

\author{
Gerald Hartig, Sabrina Gehringer und Helmut Flachberger \\ Lehrstuhl für Aufbereitung und Veredlung, Montanuniversität Leoben, Leoben, Österreich \\ Eingegangen 13. Juli 2021; angenommen 14. Juli 2021; online publiziert 10. August 2021
}

\begin{abstract}
Zusammenfassung: Einige namhafte österreichische Industrieunternehmen haben im Vorjahr eine gemeinsame Forschungsinitiative zum Thema "Methan-Pyrolyse zur Wasser- und Kohlenstoffgewinnung" gestartet und die Montanuniversität Leoben als Forschungspartner eingebunden. Parallel dazu hat das Rektorat der Montanuniversität Leoben die Forschungskapazitäten vieler Lehrstühle der alma mater Leobiensis rund um dieses Thema gebündelt.

Dem Lehrstuhl für Aufbereitung und Veredlung fällt dabei die Aufgabe zu, den im Pyrolyse-Prozess prozesstechnisch anfallenden kohlenstoffhaltigen Wertstoff zu KohlenstoffProdukten von abnahmesichernden Qualitäten zu verarbeiten. Im Zentrum der Forschungsbemühungen stehen zum einen die möglichst vollständige Abreicherung von Katalysator-Rückständen und weiteren Verunreinigungen und zum anderen die Bereitstellung von Produkten mit definierten spezifischen Oberflächen für vielfältige (industrielle) Anwendungen.
\end{abstract}

Schlüsselwörter: Methan-Pyrolyse, Aufbereitung und Veredlung, Nebenprodukt der Wasserstoff-Produktion, Kohlenstoff, Zusatzprodukt aus der WasserstoffProduktion
Processing and Refinement of Carbon Pre-concentrates from Pyrolysis Processes to Marketable Carbon Products with Regard to Large Surface Areas and High Purities-Presentation of a PhD-Project

Abstract: In the previous year, several well-known Austrian industrial companies started a joint research initiative on the topic of "methane pyrolysis for hydrogen and carbon production" and involved the Montanuniversitaet Leoben as a research partner. In parallel, the rectorate of the Montanuniversitaet Leoben has bundled the research capacities of many chairs of the alma mater Leobiensis around this topic.

The Chair of Mineral Processing is tasked with processing the carbon-containing additional product of the pyrolysis process into carbon concentrates of qualities that ensure industry acceptance. Research efforts focus on the complete removal of catalyst residues and other impurities on the one hand, and on the provision of defined specific surfaces for a wide range of (industrial) applications on the other.

Keywords: Methane pyrolysis, Mineral processing, Byproduct of hydrogen production, Carbon, Additional product of hydrogen production

\section{Einleitung}

Aufgrund der ambitionierten politischen Rahmenbedingungen zur Erreichung der Klimaneutralität in Österreich bis 2040 [1] und in der EU bis 2050 [2] wird es vielfältiger Kraftanstrengungen und fachübergreifender Forschungsinitiativen bedürfen. Insbesondere die energieintensive 
Grundstoffindustrie kann hier maßgebliche Akzente zur $\mathrm{CO}_{2}$-Reduktion setzen. So wird beispielsweise in der Metallurgie intensiv daran gearbeitet und geforscht, das Reduktionsmittel "Kohlenstoff“ durch "Wasserstoff“ zu ersetzen, um prozessbedingte $\mathrm{CO}_{2}$-Emissionen zu vermeiden. Als weiteres Beispiel sei Airbus S.A.S. angeführt, das Mitte 2020 mit ZEROe ein Konzept für mit Wasserstoff anstelle von Kerosin betriebene Flugzeuge vorgestellt hat [3].

Noch im Jahr 2010 wurde ein Großteil, nämlich $96 \%$ [4], der globalen Wasserstoff-Produktion durch Einsatz fossiler Brennstoffe erzeugt. Die Produktion erfolgt insbesondere durch Dampfreformierung (1) in Kombination mit der Wassergas-Shift-Reaktion (2). Dieses Wasserstoff-Produkt wird aufgrund der $\mathrm{CO}_{2}$-Bilanz auch als "grauer Wasserstoff" bezeichnet, im weltweiten Durchschnitt fallen hierbei etwa $12 \mathrm{~kg} \mathrm{CO} 2$ pro kg erzeugtem $\mathrm{H}_{2}$ an [5].

$$
\begin{aligned}
& \mathrm{CH}_{4(\mathrm{~g})}+\mathrm{H}_{2} \mathrm{O}_{(\mathrm{g})} \rightleftharpoons \mathrm{CO}_{(\mathrm{g})}+3 \mathrm{H}_{2(\mathrm{~g})} \\
& \mathrm{CO}_{(\mathrm{g})}+\mathrm{H}_{2} \mathrm{O}_{(\mathrm{g})} \rightleftharpoons \mathrm{CO}_{2(\mathrm{~g})}+\mathrm{H}_{2(\mathrm{~g})}
\end{aligned}
$$

Die Zukunft gehört allerdings anderen Herstellungsverfahren, an denen derzeit intensiv geforscht wird. Zwei Verfahren stehen im Fokus, nämlich die Produktion von Wasserstoff mittels Elektrolyse von Wasser ("grüner Wasserstoff“) und mittels Pyrolyse („türkiser Wasserstoff“) gemäß den nachstehenden Formeln (3) und (4). Während bei der Elektrolyse aus Wasser als Nebenprodukt "Sauerstoff $\left[\mathrm{O}_{2(\mathrm{~g})}\right]^{\prime \prime}$ (3) gebildet wird, fällt bei der Pyrolyse fester, in der Regel fein- bis feinstdisperser "Kohlenstoff $\left[\mathrm{C}_{(\mathrm{s})}\right]$ " (4) als Zusatzprodukt an. Beide Verfahren befinden sich aktuell in der Entwicklung und sind entweder aufgrund des Entwicklungsstandes noch nicht im industriellen Maßstab anwendbar oder aufgrund der Kosten noch nicht konkurrenzfähig.

$$
\begin{aligned}
& 2 \mathrm{H}_{2} \mathrm{O}_{(\mathrm{l})} \rightleftharpoons 2 \mathrm{H}_{2(\mathrm{~g})}+\mathrm{O}_{2(\mathrm{~g})} \\
& \mathrm{CH}_{4(\mathrm{~g})} \rightleftharpoons 2 \mathrm{H}_{2(\mathrm{~g})}+\mathrm{C}_{(\mathrm{s})}
\end{aligned}
$$

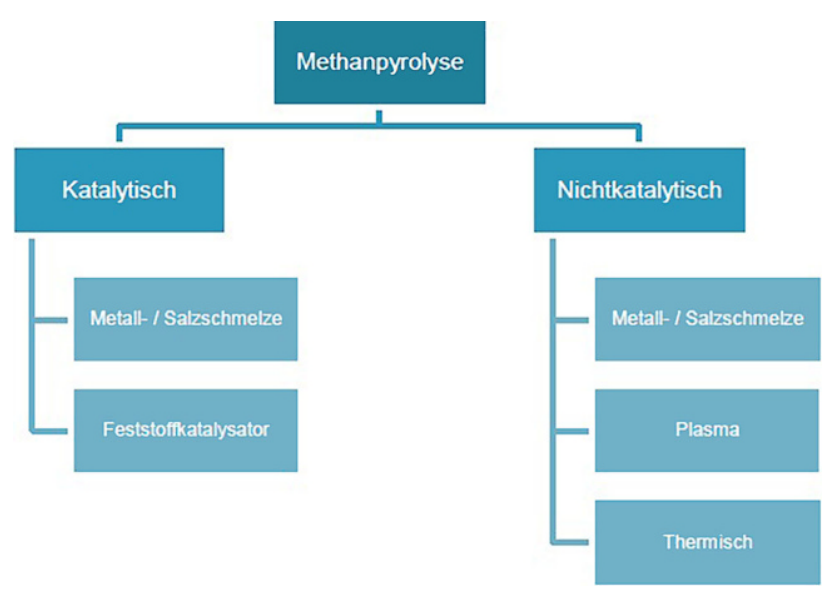

Abb. 1: Grobe Einteilung der Verfahren der Methan-Pyrolyse [13]

Zwei von namhaften österreichischen Industrieunternehmen wie auch vom Rektorat der Montanuniversität Leoben initiierte Forschungsinitiativen widmen sich schwerpunktmäßig der "türkisen“ Verfahrensroute, der "MethanPyrolyse zur Wasserstoffgewinnung."

\section{Kurzabriss zum Entwicklungsstand der Methan-Pyrolyse}

Bereits seit Beginn des 20. Jahrhunderts wird auf dem Gebiet der Methan-Pyrolyse geforscht [6, 7]. Eine deutliche Verbesserung, insbesondere in Bezug auf die Bereitstellung quantitativ belastbarer Daten, ermöglichte die Einführung der Gaschromatographie in den 1950er-Jahren [8]. Während der Fokus der Forschung lange Zeit auf dem Kohlenstoffprodukt lag, verschiebt sich dieser aufgrund der Herausforderungen der Klimakrise immer weiter in Richtung Wasserstoff.

Bis heute wird an mehreren Verfahren geforscht, eine grobe Einteilung liefert Abb. 1. Der Großteil befindet sich

\begin{tabular}{|llll}
\hline $\begin{array}{l}\text { TABELLE } 1 \\
\text { Kohlenstoffformen, ihre Anwendungsgebiete, jährlichen globalen Produktionsmengen und theoretisches Was- } \\
\text { serstoff Produktionspotenzial }\end{array}$ \\
\hline Form des Kohlenstoffs & Beispiele für Anwendungsgebiete & $\begin{array}{l}\text { Produktions- } \\
\text { menge }\end{array}$ & $\begin{array}{l}\text { Entsprechende } \mathrm{H}_{2} \\
\text { Produktion }^{\mathrm{a}}\end{array}$ \\
\hline $\begin{array}{l}\text { Industrieruß (Carbon Black) } \\
{[17]^{\mathrm{b}}}\end{array}$ & $\begin{array}{l}\text { Reifen, Druckerfarben, Hochleistungsbeschichtungen und } \\
\text { Kunststoffe }\end{array}$ & $\begin{array}{l}16,8 \mathrm{M} \text { MT } \\
(2019)\end{array}$ & $5,6 \mathrm{M}$ MT (2019) \\
\hline Graphit: & & & \\
\hline Natürlich [18, 19] & $\begin{array}{l}\text { Batterien, Schmierstoffe, Feuerfest Werkstoffe, Stahlerzeu- } \\
\text { gung }\end{array}$ & $\begin{array}{l}1,1 \mathrm{M} \text { MT } \\
(2019)\end{array}$ & $0,37 \mathrm{M}$ MT (2019) \\
\hline Synthetisch [20] & Elektroden, Stahlerzeugung, Batterien & $\begin{array}{l}1,2 \mathrm{M} \text { MT } \\
(2016)\end{array}$ & $0,4 \mathrm{M}$ MT (2016) \\
\hline $\begin{array}{l}\text { Kohlenstofffasern/ } \\
\text { Karbonfasern (Carbon fiber, } \\
\text { CF) [21] }\end{array}$ & $\begin{array}{l}\text { Luft- und Raumfahrt, Kraftfahrzeuge, Sport und Freizeit, } \\
\text { Baugewerbe, Wind Turbinen, Kohlenstoffverstärkte Kunst- } \\
\text { stoffe, Textilien }\end{array}$ & $\begin{array}{l}151 \mathrm{~K} \mathrm{MT}^{\mathrm{c}} \\
(2019)\end{array}$ & $50 \mathrm{~K}$ MT (2019) \\
\hline
\end{tabular}

$K$ Tausend, $M$ Millionen, MT metrische Tonnen

${ }^{a}$ Basierend auf dem stöchiometrischen Verhältnis von Kohlenstoff zu Wasserstoff in $\mathrm{CH}_{4}$

${ }^{\mathrm{b}}$ Die Quelle bezieht sich auf Daten der IRSG (International Rubber Study Group) die den Autoren nicht zur Verfügung stand ${ }^{c}$ Produktionskapazität 
Abb. 2: Morphologie der Kohlenstoff-Vorkonzentrate aus diversen Pyrolyse-Prozessen in Abhängigkeit von Temperatur und Katalysator [16]

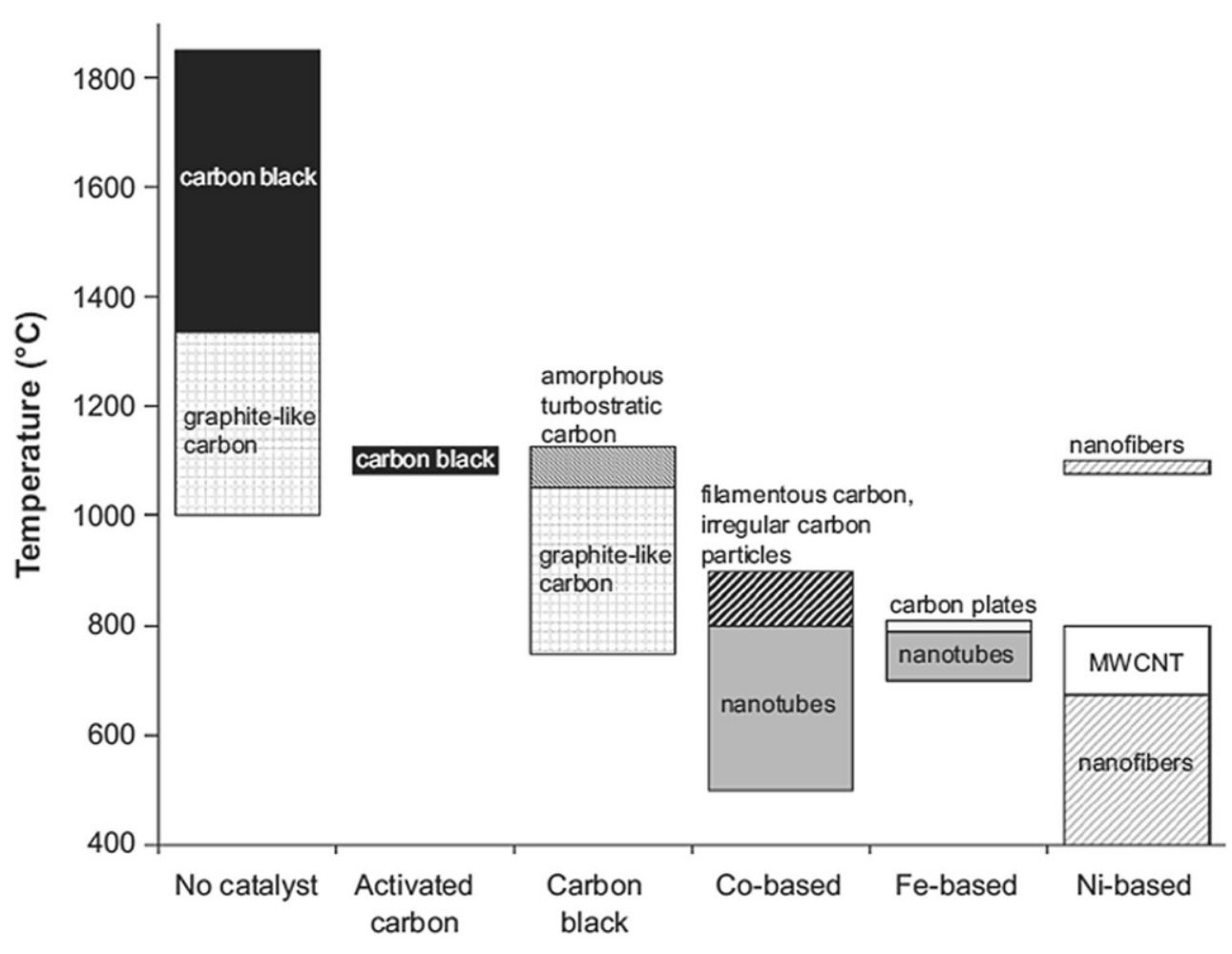

auf einem TRL (Technology Readiness Level) von 3-4 [9]. Einzelne Verfahren sind weiter fortgeschritten, das Kværner-Verfahren erreichte mit einem Plasma-basierten Pyrolyse-Prozess bereits TRL 8 und wurde durch Monolith Materials weiterentwickelt. Monolith Materials plant, im Jahr 2021 TRL 9 zu erreichen und neben $600 \mathrm{~kg} / \mathrm{h} \mathrm{H}$ eine Menge von 14.000t Carbon Black pro Jahr zu erzeugen [10]. Ebenfalls zu erwähnen ist die Firma Carbotopia aus Wien, die bereits eine kontinuierliche Pilot-Anlage errichten konnte [11] und sich auf $T R L \geq 6$ befindet [12].

\section{Kohlenstoff aus der Methan-Pyrolyse}

Wie in der Natur bilden sich unter Einfluss der Umgebungsbedingungen auch bei der Methan-Pyrolyse diverse allotrope Kohlenstoff-Formen aus. In Abhängigkeit von Katalysator und Temperatur werden Kohlenstoff-Vorkonzentrate gebildet, die in Abb. 2 einander vergleichend gegenübergestellt werden. Tab. 1, welche auf Basis einer Tabelle von Dagle et al. in [14] angefertigt wurde, stellt die unterschiedlichen Kohlenstoff-Produkte, die weltweite Produktionsmenge pro Jahr sowie die daraus zu produzierende Wasserstoffmenge dar. Sollte die Methan-Pyrolyse einen signifikanten Beitrag an der beständig steigenden weltweiten Wasserstoffproduktion (117 Mio. t [15]) leisten, müssen neue sinnstiftende Anwendungsgebiete für den anfallenden Kohlenstoff erschlossen werden.

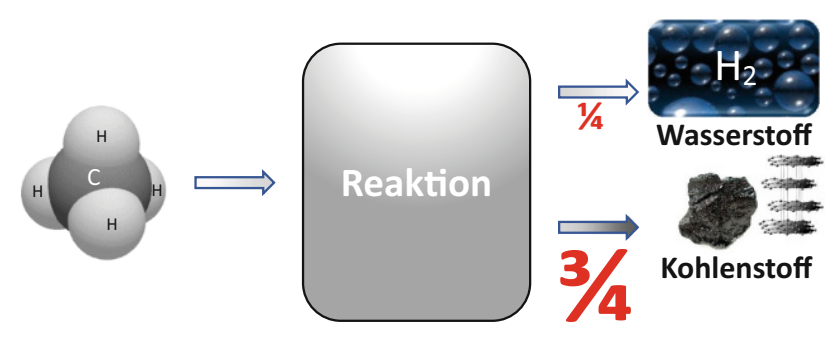

Abb. 3: Vereinfachte schaubildliche Darstellung der bei der Methan-Pyrolyse ablaufenden Reaktion ([22-25] in Anlehnung an interne Dokumentation, Projektbesprechung, Montanuniversität Leoben, vom 29.05.2020)

\section{Motivation aus aufbereitungstechnischer Sicht}

Wie auch immer die verfahrenstechnische Umsetzung der in Abb. 3 vereinfacht dargestellten Reaktion der MethanPyrolyse ausgestaltet werden wird, fallen im Zuge der $\mathrm{H}_{2}$ Produktion erhebliche Mengen an prozesstechnisch unvermeidbaren Kohlenstoff-Vorkonzentraten an.

Ganz im Sinne des "Zero Waste"-Gedankens bedarf es also in Ergänzung zu den Bemühungen zur Erzeugung qualitativ hochwertiger $\mathrm{H}_{2}$-Produkte auch einer Schwerpunktsetzung auf die möglichst vollständige Nutzung der anfallenden Kohlenstoff-Vorkonzentrate, die - und auch das geht aus der Abb. 3 eindrucksvoll hervor - mengenmäßig dominieren.

Einer möglichst vollständigen Verwertung der anfallenden Kohlenstoff-Produkte, es handelt sich um drei Tonnen pro produzierter Tonne Wasserstoff, kommt dabei eine entscheidende Rolle zu, um Wirtschaftlichkeit und Akzeptanz des Verfahrens wesentlich zu verbessern. 
Die bisherigen Untersuchungen haben aufgezeigt, dass die anfallenden Kohlenstoff-Vorkonzentrate - je nachdem, welche pyrolytischen Prozesse eingesetzt werden - einer gezielten Aufbereitung und allfälliger Veredlungsschritte bedürfen, um daraus qualitativ hochwertige KohlenstoffProdukte mit definierten Produkt-Spezifikationen zu erzeugen.

\section{Herangehensweise}

Auf Basis umfangreicher Erfahrungen zur Charakterisierung, Aufbereitung und Veredlung von natürlichen und synthetischen Grafiten sowie anderer Kohlenstoffträger hat sich der Lehrstuhl für Aufbereitung und Veredlung dieses Themas angenommen und folgendes grobes Arbeitsprogramm skizziert:

1. Erhebung/Evaluierung der geforderten Produkt-Spezifikationen für die verschiedenen Kohlenstoff-Anwendungen.

2. Erarbeitung und Festlegung einer aufbereitungstechnischen Strategie zum Erreichen der geforderten ProduktSpezifikationen.

3. Durchführung umfassender und systematischer Aufbereitungsversuche (Sortierversuche).

4. Durchführung umfassender und systematischer Versuche zur mechanischen, chemischen und thermischen Nachbehandlung für die Herstellung definierter und hoher (innerer) Oberflächen als wesentliches Qualitätskriterium für hochwertige Kohlenstoff-Produkte.

5. Zuordnung der erzeugten Kohlenstoff-Produkte in eine "Anwendungs-Datenbank", um den Rohgutwert zu maximieren und um schnell und effizient verschiedene Verwendungsmöglichkeiten von Kohlenstoff-Produkten unterschiedlicher Qualität aufzeigen zu können.

Darüber hinaus hat sich der Lehrstuhl für Aufbereitung und Veredlung auch apparativ verstärkt. Um die Bewertung und Vergleichbarkeit der Vorkonzentrate sowie der Produkte einzelner Aufbereitungsprozesse zu verbessern, wurde ein erster Schwerpunkt auf die Erweiterung der Analysetechnik gelegt. Zur Bestimmung der relevanten Kenngrößen, Kohlenstoff-Gehalt und spezifische Oberfläche, wurde der "LECO SC 832-MC" Schwefel-Kohlenstoff-Analysator von LECO Corporation und das Gemini V Oberfächenmessgerät von Micromeritics Instrument Corporation angeschafft.

\section{Erste Erkenntnisse}

Folgende Herausforderungen haben sich bislang abgezeichnet und die vorläufige Auswahl des Sortierverfahrens bestimmt:

- Die bei der Methan-Pyrolyse erzeugten Kohlenstoff-Vorkonzentrate fallen zum einen in großen Mengen und zum anderen in feinst- und polydispersen Körnerkollektiven an

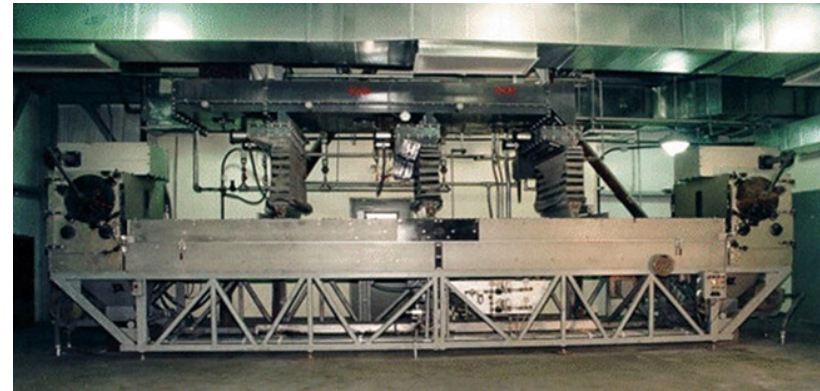

Abb. 4: Vorderansicht eines triboelektrostatischen Bandscheiders vom Hersteller ST Equipment \& Technology LLC im Pilotmaßstab [26]

\section{TABELLE 2}

Bilanztafel des Besten, auf Basis des statistischen Versuchsplans vom Typ $2^{5-1}$, durchgeführten Sortierversuches

\begin{tabular}{|llll}
\hline Produkt & $\begin{array}{l}\text { Masse- } \\
\text { ausbringen }\end{array}$ & $\begin{array}{l}\text { Kohlenstoff- } \\
\text { gehalt }\end{array}$ & $\begin{array}{l}\text { Inhalts- } \\
\text { ausbringen }\end{array}$ \\
\hline$[\%]$ & {$[\%]$} & {$[\%]$} \\
\hline Konzentrat & 96,1 & 95,0 & 97,0 \\
\hline Berge & 3,9 & 72,0 & 3,0 \\
\hline Aufgabe & 100,0 & 94,1 & 100,0 \\
\hline
\end{tabular}

- Es werden höchste Anforderungen an die Reinheit der Kohlenstoff-Produkte gestellt (z. B. vollständige Metallentfrachtung).

Im Zuge erster systematischer Versuchsreihen zur Erzeugung hochwertiger Kohlenstoff-Produkte aus Vorkonzentraten der Methan-Pyrolyse wurde die Möglichkeit einer Sortierung in trockener Betriebsweise, umgesetzt mittels Elektroscheidung, untersucht. Aufgrund der prozessbedingten Feinheit der Kohlenstoff-Vorkonzentrate $(<100 \mu \mathrm{m})$ wurde ein triboelektrostatischer Bandscheider im Labormaßstab eingesetzt. Dieser weltweit einzigartige LaborElektroscheider vom Hersteller ST Equipment \& Technology LLC ermöglicht die trockene Sortierung solch feinkörniger Kohlenstoff-Vorkonzentrate.

Die Vorderansicht eines Bandscheiders im Pilotmaßstab ist in Abb. 4 ersichtlich.

Die Sortierversuche am triboelektrostatischen Bandscheider wurden auf Basis eines statistischen Versuchsplans (Typ $2^{5-1}$ ) abgewickelt, um effizient möglichst viele Einstellparameter und deren Wechselwirkung zueinander zu untersuchen. Der eingesetzte Versuchsplan hat sich bereits für die Sortierung zahlreicher Rohgutproben am triboelektrostatischen Bandscheider bewährt.

Im Zuge dieser Sortierversuche konnte eine eindeutige Anreicherung des Kohlenstoffs und eine Abreicherung der Begleitbestandteile im Konzentrat erzielt werden. Die Bilanztafel zum besten Versuch ist in Tab. 2 dargestellt. Bei diesem einstufigen Versuch konnte der Kohlenstoffgehalt im Konzentrat, verglichen mit der Aufgabe, um 0,9\%Punkte gesteigert werden. Dabei wurde ein Kohlenstoffgehalt von $95,0 \%$, bei einem Inhaltsausbringen von $97,0 \%$, erreicht. 
Im Zuge dieser orientierenden Untersuchungen zur Aufbereitung von Kohlenstoff aus der Methan-Pyrolyse mittels elektrostatischer Sortierung konnten somit An- und Abreicherungseffekte erkannt werden. Zur Auffindung von optimierten Sortierlösungen, abgestimmt auf die große Bandbreite unterschiedlicher Kohlenstoff-Vorkonzentrate aus der Methan-Pyrolyse, bedarf es aber noch intensiver Forschungstätigkeit, welche im Rahmen des, in diesem Artikel vorgestellten Dissertationsprojekts realisiert werden soll.

Funding. Open access funding provided by Montanuniversität Leoben.

Open Access Dieser Artikel wird unter der Creative Commons Namensnennung 4.0 International Lizenz veröffentlicht, welche die Nutzung, Vervielfältigung, Bearbeitung, Verbreitung und Wiedergabe in jeglichem Medium und Format erlaubt, sofern Sie den/die ursprünglichen Autor(en) und die Quelle ordnungsgemäß nennen, einen Link zur Creative Commons Lizenz beifügen und angeben, ob Änderungen vorgenommen wurden.

Die in diesem Artikel enthaltenen Bilder und sonstiges Drittmaterial unterliegen ebenfalls der genannten Creative Commons Lizenz, sofern sich aus der Abbildungslegende nichts anderes ergibt. Sofern das betreffende Material nicht unter der genannten Creative Commons Lizenz steht und die betreffende Handlung nicht nach gesetzlichen Vorschriften erlaubt ist, ist für die oben aufgeführten Weiterverwendungen des Materials die Einwilligung des jeweiligen Rechteinhabers einzuholen.

Weitere Details zur Lizenz entnehmen Sie bitte der Lizenzinformation auf http://creativecommons.org/licenses/by/4.0/deed.de.

\section{Literatur}

1. Die neue Volkspartei, Die Grünen - Die Grüne Alternative (2020) Bundeskanzleramt Österreich, Aus Verantwortung für Österreich. Regierungsprogramm 2020-2024, Wien, S. 72, Text abrufbar unter: https://www.bundeskanzleramt.gv.at/bundeskanzleramt/diebundesregierung/regierungsdokumente.html (23.06.2021)

2. General Secretariat of the Council (5. Mai 2021): Proposal for a Regulation of the European Parliament and of the Council establishing the framework for achieving climate neutrality and amending Regulation (EU) 2018/1999 (European Climate Law), Brüssel, S. 4, Text abrufbar unter: https://data.consilium.europa.eu/doc/document/ST8440-2021-INIT/en/pdf (23.06.2021)

3. Airbus S.A.S: ZEROe Towards the world's first zero-emission commercial aircraft https://www.airbus.com/innovation/zero-emission/ hydrogen/zeroe.html (16.06.2021)

4. Töpler, J.; Lehmann, J.: Hydrogen and Fuel Cell Technologies and Market Perspectives, S. 5, Berlin: Springer, 2016

5. Fulcheri, L.: Methane pyrolysis: The third way for low $\mathrm{CO}_{2}$ hydrogen [Debate presentation] \#FSRDebates, online, 2021 https://fsr.eui. eu/event/pyrolysis-hydrogen-truly-neutral-or-negative-will-it-becompetitive/ (23.06.2021)

6. Bone, W. A.; Coward, H. F.: The Thermal Decomposition of Hydrocarbons. Part I. [Methane, Ethane, Ethylene, and Acetylene.] Journal of the Chemical Society, Transactions, 93 (1908), pp 1197-1225

7. Slater, W. E.: The influence of different surfaces on the decomposition of methane, Journal of the Chemical Society, Transactions, 109 (1916), pp 160-164

8. Fau, G.; Gascoin, N.; Gillard, P.; Steelant, J.: Methane Pyrolysis: literature survey and comparisons of available data for use in numerical simulations, Journal of Analytical and Applied Pyrolysis, 104 (2013), pp 1-9
9. Schneider, S.; Bajohr, S.; Graf, F.; Kolb, T.: Verfahrensübersicht zur Erzeugung von Wasserstoff durch Erdgas-Pyrolyse, Chemie Ingenieur Technik, WILEY-VCH, 92 (2020), No. 8, pp 1023-1032

10. Mesrobian, C.: Taking Methane Pyrolysis from Concept to Industrial Plant, Monolith Materials, 2021, https://arpa-e.energy. gov/sites/default/files/2021-01/08\%200K\%20-Monolith_ARPAE_ MethanePyrolysis2021_v3.pdf (24.06.2021)

11. Mauthner, K.: Entwicklung einer kontinuierlichen Wasserstoff-Carbon Nanotubes Demo-Produktionsanlage, Wien: Bundesministerium für Verkehr, Innovation und Technologie, 2011

12. Petters, S.: FSR online workshop Very-low / decarbonised hydrogen from natural gas, https://fsr.eui.eu/event/very-low-decarbonisedhydrogen-from-natural-gas/ (24.06.2021)

13. Weiss, H.: Stand des Wissens und Orientierungsversuche zur Methanpyrolyse, Masterarb., Leoben, Montanuniv., Lehrstuhl für Verfahrenstechnik des industriellen Umweltschutzes, 2021

14. Dagle, R.; Dagle, V.; Bearden, M.; Holladay, J.; Krause, T.; Ahmed S.: R\&D Opportunities for Development of Natural Gas Conversion Technologies for Co-Production of Hydrogen and Value-Added Solid Carbon Products, ANL-17/11, PNNL-26726, National Technical Information Service, Alexandria (VA), USA, 2017

15. IEA: The Future of Hydrogen Seizing today's opportunities, Report prepared by the IEA for the G20, Japan, IEA Publications, 2019, https://iea.blob.core.windows.net/assets/9e3a3493-b9a6-4b7d-b4997ca48e357561/The_Future_of_Hydrogen.pdf (25.06.2021)

16. Keipi, T.; Tolvanen, K.E.S.; Tolvanen, H.; Konttinen, J.: Thermo-catalytic decomposition of methane: The effect of reaction parameters on process design and the utilization possibilities of the produced carbon, Energy Conversion and Management, 126 (2016), pp 923-934

17. Mordor Intelligence: Carbon Black Market-Growth, Trends, COVID-19 Impact, and Forecasts (2021-2026) https://www. mordorintelligence.com/industry-reports/carbon-black-market (23.06.2021)

18. Reichl, C.; Schatz M.: World Mining Data 2021 Volume 36, Wien: Bundesministerium für Landwirtschaft, Regionen und Tourismus, 2021

19. Olsen D. W.: GRAPHITE (NATURAL), U.S. Geological Survey, Mineral Commodity Summaries, 2020, https://pubs.usgs.gov/ periodicals/mcs2020/mcs2020-graphite.pdf (23.06.2021)

20. More, A.: Synthetic Graphite Market 2021 Growth Analysis, Share and consumption by Regional data, Investigation and Growth, Demand by Regions, Types and Analysis of Key Players-Research Forecasts To 2027 with Top Growth Companies, https:// www.marketwatch.com/press-release/synthetic-graphite-market2021-growth-analysis-share-and-consumption-by-regional-datainvestigation-and-growth-demand-by-regions-types-and-analysisof-key-players--research-forecasts-to-2027-with-top-growthcompanies-2021-03-28 (23.06.2021)

21. Sauer, M.: Composites-Marktbericht 2019-Der globale CF- und CC-Markt 2019 - Marktentwicklungen, Trends, Ausblicke und Herausforderungen-veröffentlichte Kurzfassung, https://compositesunited.com/media/3989/ger_ccev_marktbericht_2019_kurzversion. pdf (23.06.2021)

22. https://commons.wikimedia.org/w/index.php?title=File:3D_methane. PNG\&oldid=483619216 (01.07.2021)

23. https://commons.wikimedia.org/w/index.php?title=File:Graphitetn 19a.jpg\&oldid $=504798758(01.07 .2021)$

24. https://commons.wikimedia.org/w/index.php?title=File:Graphenegraphite relation.png\&oldid $=508543594$ (01.07.2021)

25. https://commons.wikimedia.org/w/index.php?title=File:Bubbles_in_ the_dark.jpg\&oldid=468030016 (01.07.2021)

26 Dötterl, M. et al.: Electrostatic separation, Ullmann's Encyclopedia of Industrial Chemistry, Weinheim: WILEY-VCH, 2016

Hinweis des Verlags. Der Verlag bleibt in Hinblick auf geografische Zuordnungen und Gebietsbezeichnungen in veröffentlichten Karten und Institutsadressen neutral. 